\title{
A Phase I Study of Carfilzomib for Relapsed or Refractory Acute Myeloid and Acute Lymphoblastic Leukemia
}

\author{
Lukas D. Wartman, MD¹, Mark A. Fiala, BS, CCRP ${ }^{1}$, Theresa Fletcher, MS ${ }^{1}$, Emily R. \\ Hawkins, BS, CCRP ${ }^{1}$, Amanda Cashen, MD ${ }^{1}$, John F. DiPersio, MD, PhD ${ }^{1}$, Meagan A. \\ Jacoby, MD ${ }^{1}$, Keith E. Stockerl-Goldstein, MD ${ }^{1}$, Iskra Pusic, MD ${ }^{1}$, Geoffrey L. Uy, MD ${ }^{1}$, Peter \\ Westervelt, MD, PhD ${ }^{1}$, and Ravi Vij, MD, MBBS ${ }^{1}$ \\ ${ }^{1}$ Division of Oncology, Department of Medicine, Washington University School of Medicine, St. \\ Louis MO
}

The outcome for adult patients with acute myeloid leukemia (AML) and acute lymphoblastic leukemia (ALL) remains poor; new chemotherapy agents with activity against these leukemias are urgently needed. Proteasome inhibition has established efficacy in several other hematologic malignancies and is a promising therapeutic target in acute leukemias $\left[{ }^{1},{ }^{2}\right]$. Multiple trials have demonstrated that the proteasome inhibitor bortezomib has encouraging clinical activity in the treatment of acute leukemias, notably when combined with other cytotoxic chemotherapy, whereas little anti-leukemic activity was seen when it was used as a single agent $\left[{ }^{3}\right]\left[{ }^{4}\right]\left[\left[^{5}\right]\left[{ }^{6}\right]\left[{ }^{7}\right]\right.$. The second-generation proteasome inhibitor carfilzomib (CFZ) has also demonstrated potent in vitro anti-leukemic activity) [ $\left.{ }^{8}\right]$ [ ${ }^{9}$. Given the above findings and the demonstrated efficacy and safety profile of CFZ in the treatment of multiple myeloma, there was a strong rationale to begin the clinical study of this agent in the treatment of acute leukemias $\left[{ }^{10}\right]$. Therefore, we performed the first phase I, single arm, single center, open label study of CFZ in adult patients with relapsed or refractory AML and ALL to determine the safety and tolerability of CFZ in this patient population.

The primary objective was to determine the maximum tolerated dose (MTD) and doselimiting toxicity (DLT) of CFZ in adult patients with relapsed/refractory AML and ALL. Secondary objectives included: determination of disease response (according to the International Working Group Criteria for AML, adapted for ALL patients so response criteria were uniform), event-free survival (EFS), and overall survival (OS) $\left[{ }^{11}\right]$. This was a $3+3$ dose escalation phase I study. The Washington University Institutional Review Board (IRB) approved the study, and all patients signed an informed consent prior to enrollment. The study was conducted in compliance with the Declaration of Helsinki, and the applicable local and national regulations. The clinical trial was registered at www.clinicaltrials.gov as NCT01137747.

Corresponding author: Lukas D. Wartman, MD, Division of Oncology, Department of Medicine, Washington University School of Medicine, 660 S. Euclid Avenue, Campus Box 8007, St. Louis MO 63110, USA, Phone: 314-454-8304, Fax: 314-362-3165, ; Email: lwartman@dom.wustl.edu 
Patients with relapsed or refractory AML or ALL (documented on bone marrow biopsy done within 14 days of starting cycle 1) following one or more lines of therapy were included. Eligible patients were required to be at least 18 years old, have a ECOG performance status of 2 or below, and have adequate organ function including: creatinine clearance $\geq 15 \mathrm{~mL} /$ minute; total bilirubin $\leq 2.0 \mathrm{mg} / \mathrm{dL}$; alanine transaminase (ALT) $\leq 2.5 \times$ upper limit of normal (or up to $\leq 5.0 \times$ if due to leukemic involvement); and uric acid within normal limits. Patients were excluded if they had any of the following: active graft-vs-host disease; central nervous system leukemia; significant peripheral neuropathy (grades 3 and 4 or painful grade 2 neuropathy); HIV or active hepatitis A, B or C; a peripheral blast count of $\geq 30,000 / \mu \mathrm{L}$; were pregnant or nursing; or had cytotoxic chemotherapy (including investigational agents) or radiotherapy within 14 days or an allogeneic transplantation within 100 days prior to enrollment. Patients were excluded if they could not tolerate fluid hydration or had significant pleural effusions or ascites.

CFZ was administered as a 30-minute infusion on days $1,2,8,9,15,16,22$, and 23 of a 28 day cycle at escalating doses of 36,45 , and $56 \mathrm{mg} / \mathrm{m}^{2}$ based on actual calculated body surface area $\left.{ }^{12}\right]$. As relapsed acute leukemias are generally more aggressive malignancies than multiple myeloma or solid tumors, the dosing of carfilzomib was extended to days 22 and 23 in this trial. Data from carfilzomib dosing in a murine model suggests that no rest period was necessary to avoid toxicity $\left[{ }^{8}\right]$. Up to 6 cycles of treatment were allowed. All dose levels included lead-in doses of $20 \mathrm{mg} / \mathrm{m}^{2} \mathrm{CFZ}$ on days 1 and 2 of cycle 1 . For dose level 0 (starting dose level), CFZ was then increased to $27 \mathrm{mg} / \mathrm{m}^{2}$ on days 8 and 9 of cycle 1 only followed by $36 \mathrm{mg} / \mathrm{m}^{2}$ for all subsequent doses. For dose level +1 , CFZ was increased to $36 \mathrm{mg} / \mathrm{m}^{2}$ on days 8 and 9 of cycle 1 only followed by $45 \mathrm{mg} / \mathrm{m}^{2}$ for all subsequent doses. For dose level +2 , CFZ was increased to $45 \mathrm{mg} / \mathrm{m}^{2}$ on days 8 and 9 of cycle 1 only followed by $56 \mathrm{mg} / \mathrm{m}^{2}$ for all subsequent doses.

Pre-medication with dexamethasone (4 mg) was administered prior to all CFZ doses. Intravenous hydration prior to drug dosing was mandatory during cycle 1 (and continued in cycle 2 for patients at risk for tumor lysis syndrome). All patients received allopurinol prophylaxis and standard supportive care including: transfusions, antiemetics, and antibiotics according to institutional guidelines. A complete metabolic panel (CMP) and complete blood count (CBC) were reviewed prior to CFZ dosing.

MTD was defined as the highest dose-level tested in which less than 1 of 3 or 2 of 6 patients experienced a DLT. DLT was defined as any $\geq$ grade 2 neuropathy with pain, or any other grade 3-5 non-hematologic toxicity related to study treatment occurring during cycle 1 . Hematologic toxicities and infections were not considered DLTs. After 2 cycles of CFZ, the treating physician could proceed to an alternative treatment or continue the patient on study for up to 6 cycles if the patient did not have progressive disease. Blood counts were performed at least weekly while on study; bone marrow examinations were performed on day 28 of cycles 1, 2, 4 and 6 . Additional blood counts and/or bone marrow examinations were performed at the discretion of the treating physician. All patients who completed one cycle of treatment were considered evaluable for response. Toxicities were collected from the start of CFZ study treatment through 28 days following the last CFZ dose, or until start 
of a subsequent chemotherapy or death, and graded according to Version 4.0 of the National Cancer Institute Common Terminology Criteria for Adverse Events.

From December 2010 through July 2014, 18 patients were enrolled in the study. Eight patients discontinued prior to completion of cycle 1 for reasons other than study-related toxicity and were replaced. The median age at enrollment was 70 years (range 32-78), and 12 of the 18 patients were male. Seventeen patients had AML ( 7 had complex cytogenetics) while 1 patient had ALL. The median number of prior therapies was 2 (range 1-4); two patients had undergone prior allogeneic stem cell transplantation. The median time from initial diagnosis to the start of the protocol was 9.7 months (range 1.4-102.0). At study entry, median white blood cell (WBC) count was $3.2 \times 10^{9} / \mathrm{L}$ (range $0.6-42.2$ ), median peripheral blood absolute blast cell count was $0.88 \times 10^{9} / \mathrm{L}$ (range $0-22.78, \%$ peripheral blasts range $0-62 \%$ with median of $18 \%$ ), and bone marrow blast percentage was $45.5 \%$ (range 11-92\%).

Three patients were enrolled into dose level $0\left(36 \mathrm{mg} / \mathrm{m}^{2}\right)$. One patient was removed prior to completing cycle 1 due to disease progression and was replaced. None of the patients treated at dose level 0 experienced a DLT. A total of 8 patients were enrolled into dose level +1 (45 $\left.\mathrm{mg} / \mathrm{m}^{2}\right), 4$ of which were evaluable for DLT analysis. No DLTs were experienced and therefore 6 patients were enrolled into dose level $+2\left(56 \mathrm{mg} / \mathrm{m}^{2}\right)$; three of these patients were evaluable for DLT analysis. As there were no DLTs observed in the maximum dose level tested, a MTD was not established.

Ten out of the 18 patients were evaluable for disease response; 2 patients achieved a partial response (PR), and 4 additional patients had stable disease (SD). Table I summarizes the response of each patient. The median duration on study treatment was 21.5 days (range 164). The median EFS was 1.2 months (range 0.2-2.4), and the median OS was 2.3 months. As expected in this patient cohort with relapsed/refractory acute leukemia, 14 of the 18 patients enrolled experienced a serious adverse event not related to study treatment during the toxicity assessment window. The most common grade 3/4 non-hematologic toxicities regardless of attribution were: dyspnea (17\%), activated partial thromboplastin time prolongation (17\%), intracranial hemorrhage or hematoma (11\%), infection (11\%), congestive heart failure (CHF) exacerbation (11\%), and hypophosphatemia (11\%). We observed no pattern of recurrent minor toxicities or increase in toxicities with dose escalation. Both patients with CHF exacerbations had a history of underlying cardiac disease. The first patient had a similar CHF exacerbation prior to study entry, and therefore the reported event was not likely related to CFZ. The second patient had a myocardial infarction in the setting of known atherosclerotic disease and was found to have mildly reduced LV systolic function compared to study entry. Although unlikely, the CHF could have been related to CFZ.

In conclusion, we observed modest anti-leukemic activity in this limited phase 1 study of CFZ in heavily pre-treated patients with AML or ALL. The administration of CFZ was safe and well tolerated, and a MTD was not established. The recommended dosage for further studies is $56 \mathrm{mg} / \mathrm{m}^{2}$. Additional clinical trials incorporating CFZ in combination with other chemotherapeutic agents are now needed to assess the potential efficacy of CFZ in the 
treatment of AML and ALL, especially in light of recently published data supporting the use of proteasome inhibition in patients with ALL and MLL-fusions and the preferential activity of CFZ over bortezomib against AML stem cells (CD34+/CD38- cells) $\left[{ }^{13}\right]\left[{ }^{14}\right]$.

\section{References}

1. McBride A, Ryan PY. Proteasome inhibitors in the treatment of multiple myeloma. Expert Rev Anticancer Ther. 2013; 13(3):339-358. [PubMed: 23477520]

2. Niewerth D, Dingjan I, Cloos J, Jansen G, Kaspers G. Proteasome inhibitors in acute leukemia. Expert Rev Anticancer Ther. 2013; 13(3):327-337. [PubMed: 23477519]

3. Horton TM, Pati D, Plon SE, et al. A phase 1 study of the proteasome inhibitor bortezomib in pediatric patients with refractory leukemia: a Children's Oncology Group study. Clin Cancer Res. 2007; 13(5):1516-1522. [PubMed: 17332297]

4. Attar EC, De Angelo DJ, Supko JG, et al. Phase I and pharmacokinetic study of bortezomib in combination with idarubicin and cytarabine in patients with acute myelogenous leukemia. Clin Cancer Res. 2008; 14(5):1446-1454. [PubMed: 18316568]

5. Messinger Y, Gaynon P, Raetz E, et al. Phase I study of bortezomib combined with chemotherapy in children with relapsed childhood acute lymphoblastic leukemia (ALL): a report from the therapeutic advances in childhood leukemia (TACL) consortium. Pediatr Blood Cancer. 2010; 55(2):254-259. [PubMed: 20582937]

6. Messinger YH, Gaynon PS, Sposto R, et al. Bortezomib with chemotherapy is highly active in advanced B-precursor acute lymphoblastic leukemia: Therapeutic Advances in Childhood Leukemia \& Lymphoma (TACL) Study. Blood. 2012; 120(2):285-290. [PubMed: 22653976]

7. Sarlo C, Buccisano F, Maurillo L, et al. Phase II Study of Bortezomib as a Single Agent in Patients with Previously Untreated or Relapsed/Refractory Acute Myeloid Leukemia Ineligible for Intensive Therapy. Leuk Res Treatment. 2013; 2013:705714. [PubMed: 23738080]

8. Demo SD, Kirk CJ, Aujay MA, et al. Antitumor activity of PR-171, a novel irreversible inhibitor of the proteasome. Cancer Res. 2007; 67(13):6383-6391. [PubMed: 17616698]

9. Stapnes C, Doskeland AP, Hatfield K, et al. The proteasome inhibitors bortezomib and PR-171 have antiproliferative and proapoptotic effects on primary human acute myeloid leukaemia cells. $\mathrm{Br} \mathrm{J}$ Haematol. 2007; 136(6):814-828. [PubMed: 17341267]

10. Sugumar D, Keller J, Vij R. Targeted treatments for multiple myeloma: specific role of carfilzomib. Pharmgenomics Pers Med. 2015; 8:23-33. [PubMed: 25691814]

11. Cheson BD, Bennett JM, Kopecky KJ, et al. Revised recommendations of the International Working Group for Diagnosis, Standardization of Response Criteria, Treatment Outcomes, and Reporting Standards for Therapeutic Trials in Acute Myeloid Leukemia. J Clin Oncol. 2003; 21(24):4642-4649. [PubMed: 14673054]

12. Jakubowiak AJ. Evolution of carfilzomib dose and schedule in patients with multiple myeloma: a historical overview. Cancer Treat Rev. 2014; 40(6):781-790. [PubMed: 24630735]

13. Liu H, Westergard TD, Cashen A, et al. Proteasome inhibitors evoke latent tumor suppression programs in pro-B MLL leukemias through MLL-AF4. Cancer Cell. 2014; 25(4):530-542. [PubMed: 24735925]

14. van der Helm LH, Bosman MC, Schuringa JJ, Vellenga E. Effective targeting of primitive AML CD34 cells by the second-generation proteasome inhibitor carfilzomib. Br J Haematol. 2015 


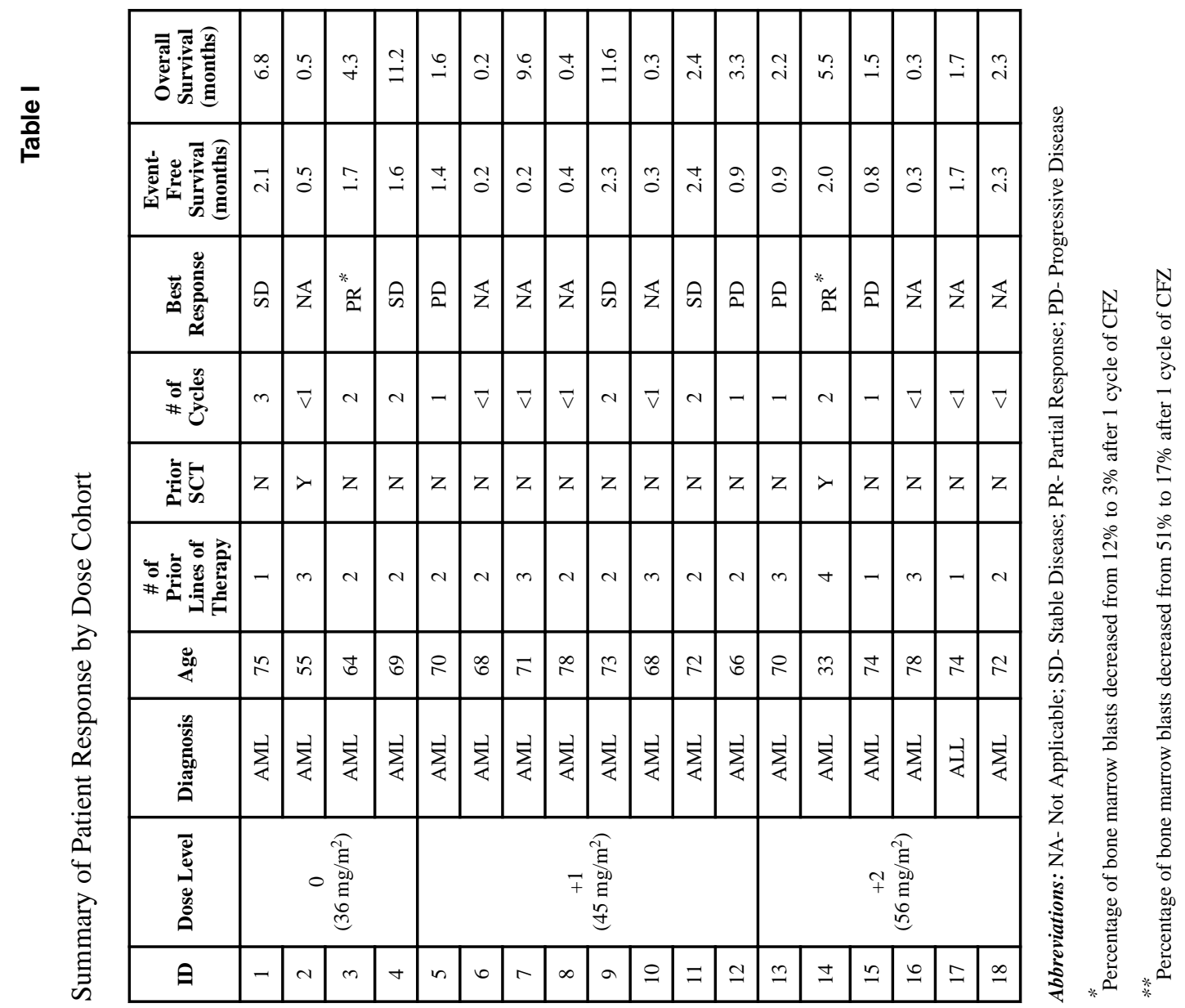

